\title{
Childhood abuse, the BDNF-Val66Met polymorphism and adult psychotic-like experiences
}

Silvia Alemany, Bárbara Arias, Mari Aguilera, Helena Villa, Jorge Moya, Manuel I. Ibáñez, Helen Vossen, Cristobal Gastó, Generós Ortet and Lourdes Fañanás

\section{Background}

The well-established relationship between childhood adversity and psychosis is likely to involve other factors such as genetic variants that can help us to understand why not everyone exposed to adverse events develops psychotic symptoms later in life.

\section{Aims}

We investigated the influence of childhood abuse and neglect on positive and negative psychotic-like experiences in adulthood and the potential moderating effect of the BDNF-Val66Met polymorphism.

\section{Method}

Psychotic-like experiences and childhood adversity were assessed in 533 individuals from the general population.

\section{Results}

Childhood abuse showed a strong independent effect on the positive dimension of psychotic-like experiences $(\beta=0.16$, s.e. $=0.05, P=0.002$ ). Furthermore, this association was moderated by the BDNF-Val66Met polymorphism ( $\beta=0.27$, s.e. $=0.10, P=0.004$ )

\section{Conclusions}

Individuals exposed to childhood abuse are more likely to report positive psychotic-like experiences. Met carriers reported more positive psychotic-like experiences when exposed to childhood abuse than did individuals carrying the $\mathrm{Val} / \mathrm{Val}$ genotype. Therefore, the observed gene-environment interaction effect may be partially responsible for individual variation in response to childhood abuse.

\section{Declaration of interest}

None.
Psychological stress occurring during either childhood or adulthood has been related to psychosis. ${ }^{1}$ Childhood adversity as a form of psychological stress has been shown to be a risk factor for the development of psychotic symptoms in clinical samples ${ }^{2,3}$ and psychotic-like experiences in individuals from the general population. ${ }^{4,5}$ Despite this established relationship, it is necessary to consider the type and severity of any environmental exposure, together with a specific individual genetic background of risk, in order to understand the development of psychosis in adulthood. The term childhood adversity usually refers to a wide range of severe adverse experiences occurring early in life (before 16 years of age) and includes sexual, physical and emotional abuse and neglect. In this regard, several lines of research have suggested a strong relationship between childhood abuse and positive psychotic symptoms. ${ }^{6}$ However, less attention has been paid to the effect of neglect, and studies examining the impact of both types of childhood adversity are still relatively scarce. Genetic factors are also likely to be involved in the individual variation in response to stress. Genes involved in regulating the adaptive behavioural response to stress represent plausible candidates to explore putative gene-environment interaction effects in the association between childhood adversity and psychotic symptoms. In this context, the BDNF-Val66Met polymorphism has been related not only to psychosis but has also been shown to moderate the impact of childhood adversity on the later expression of affective symptoms. ${ }^{7,8}$ Brain-derived neurotrophic factor (BDNF) is a neurotrophin that promotes the growth and differentiation of developing neurons in central and peripheral nervous systems. ${ }^{9}$ It has been shown that early stress can influence BDNF expression and produce long-lasting effects on neurotrophic processes, thereby having an impact on neuronal maturation and plasticity in later life. ${ }^{1}$ However, studies of the relationship between the functional BDNF-Val66Met polymorphism and schizophrenia have produced mixed results, ${ }^{9}$ which may be because of the underlying gene-environment interaction. ${ }^{10}$ One recent study found that Met carriers (negatively affecting intracellular processing and secretion of the mature protein) report more paranoid feelings in the presence of social stress than do $\mathrm{Val} / \mathrm{Val}$ carriers. ${ }^{11}$ Thus, it is plausible that the BDNF-Val66Met polymorphism might play a moderating role in the association between childhood adversity and psychosis. The present study aimed to explore whether childhood adversity (childhood abuse and childhood neglect) have a differential impact on the presence of psychotic-like experiences. Furthermore, a possible moderating effect of the BDNF-Val66Met polymorphism on the relationship between childhood adversity and psychotic-like experiences was also investigated.

\section{Method}

\section{Sample}

The sample consisted of 533 individuals who were recruited from the campus of the Jaume I University in Castello (Spain), as well as from university offices and community technical schools in the metropolitan area of Barcelona (Spain). All the participants were adults (mean age 22.9 years, s.d. $=5.4$ ) and $45.4 \%$ were males. At the time of the assessment $77 \%$ of the participants were students. Exclusion criteria were the presence of any major medical illness affecting brain function, neurological conditions and a history of head injury. All participants were of Spanish (White) ancestry, thereby reducing the possibility of confounding genetic differences by population stratification. ${ }^{12}$ Ethical approval was obtained from 
local research ethics committees. All participants provided written informed consent before inclusion in the study.

\section{Measures}

The Community Assessment of Psychic Experiences (CAPE) ${ }^{13}$ was used to assess positive and negative psychotic-like experiences in the sample. This validated self-report questionnaire measures the lifetime prevalence of psychotic-like experiences on a frequency scale ranging from 'never' to 'nearly always'. Examples of the items assessing the positive and negative dimension are, respectively, 'do you ever feel as if things in magazines or TV were written especially for you?' and 'do you ever feel that you experience few or no emotions at important events?'. The CAPE provides a total score per dimension by adding up the scores on the frequency items.

Childhood adversity was assessed by the shortened version of the Childhood Trauma Questionnaire (CTQ). ${ }^{14}$ This questionnaire consists of 28 items measuring five types of childhood trauma: emotional abuse, physical abuse, sexual abuse, emotional neglect and physical neglect. Childhood adversity was grouped into two main types: childhood abuse (including emotional, physical and sexual abuse) and childhood neglect events (including emotional and physical neglect). This was done in order to explore the putative specificity of the impact of these two types of childhood adversity. Childhood abuse and childhood neglect were calculated by summing the items included for each type of childhood adversity. Example items of childhood abuse and childhood neglect are, respectively, 'people in my family hit me so hard that it left me with bruises or marks' and 'my parents were too drunk or high to take care of the family'. The score for each item ranges from 1 to 5 ('never true' to 'very often true'), depending on the extent to which individuals agree with the statement. Reliability and validity of the CTQ have both been demonstrated. ${ }^{15}$

Because schizotypal personality, cannabis use and anxiety levels have all been related to both childhood adversity and psychotic-like experiences, and given the relationship between them, ${ }^{16-18}$ the analyses were corrected for these variables, along with age and gender as other potential confounders. Schizotypal personality was measured with the Schizotypy Personality Questionnaire-Brief (SPQ-B). ${ }^{19}$ Cannabis use was assessed with one question regarding the frequency of consumption: 'never', 'once', 'monthly', 'weekly' or 'daily' (this variable was then dichotomised into two categories: 'not exposed to cannabis': never, once; and 'exposed to cannabis': monthly, weekly, daily). Anxiety as a behavioural trait was assessed using the State-Trait Anxiety Inventory (STAI-T). ${ }^{20}$

\section{Laboratory methods}

Genomic DNA was extracted from peripheral blood cells using the Real Extraction DNA Kit (Durviz SLU, Valencia, Spain), or from buccal mucosa on a cotton swab using the BuccalAmp DNA Extraction Kit (Epicentre Biotechnologies, Madison, Wisconsin, USA). The rs6265 SNP (Val66Met) of the BDNF gene was determined using the Taqman $5^{\prime}$ exonuclease assay (Applied Biosystems) and genotyped using Applied Biosystems (AB) TaqMan technology. The probe for genotyping the rs6265 was ordered through the TaqMan SNP Genotyping assays (code C_11592758_10) AB assay-on-demand service. The final volume of the polymerase chain reaction was $5 \mathrm{ml}$, which contained $10 \mathrm{ng}$ of genomic DNA, $2.5 \mathrm{ml}$ of TaqMan Master Mix, and $0.125 \mathrm{ml}$ of $40 \mathrm{x}$ genotyping assay. The cycling parameters were as follows: $95^{\circ} \mathrm{C}$ for $10 \mathrm{~min}$ followed by 40 cycles of denaturation at $92^{\circ} \mathrm{C}$ for $15 \mathrm{~s}$ and annealing/extension at $60^{\circ} \mathrm{C}$ for 1 min. Polymerase chain reaction plates were read on an $\mathrm{ABI}$
PRISM 7900HT instrument with SDS v2.1 software (Applied Biosystems).

\section{Statistical analyses}

Multiple linear regressions were conducted using STATA 10.0 for Windows. Separate models were tested for CAPE positive and CAPE negative as dependent variables. For the first hypothesis the independent variables of interest were childhood abuse, childhood neglect and the BDNF-Val66Met polymorphism. Schizotypal personality, cannabis use, trait anxiety, gender and age were included in the model as covariates. For the second hypothesis, two-way interaction effects between childhood abuse and the BDNF-Val66Met polymorphism and childhood neglect and the BDNF-Val66Met polymorphism were added to the model, as described for the first hypothesis. Since the Met/Met genotype $(n=29)$ has a much lower frequency than the Val/Met and $\mathrm{Val} / \mathrm{Val}$ genotypes, the genotypes for this polymorphism were included in the analyses as a binary variable (Met allele carriers and Val homozygotes).

\section{Results}

\section{Descriptive statistics}

In order to obtain the prevalence of psychotic-like experiences in the current sample, CAPE scores were recoded to 0 (never, sometimes) and 1 (often, almost always). The resulting prevalence rate indicated that psychotic-like experiences were quite frequent. Specifically, $40.7 \%$ of the sample often or almost always experienced at least one positive psychotic-like experience; similarly, $47.6 \%$ reported experiencing at least one negative psychotic-like experience often or almost always.

The prevalence of childhood adversity was evaluated by recoding the answers to 0 (never true) and 1 (rarely true, sometimes true, often true and very often true). Thus, 1 indicates that the individual was exposed at least once to the adverse event. In the current sample, $25.5 \%$ of the individuals were exposed to childhood abuse and $32.2 \%$ to childhood neglect. More details of the distribution of dimensions in the sample can be found elsewhere. ${ }^{8}$

Genotype information was available for 470 individuals. The genotype frequencies for the BDNF-Val66Met polymorphism were: $\mathrm{Val} / \mathrm{Val}: \quad 60 \%(n=282)$; Val/Met: $33.8 \% \quad(n=159)$; and Met/Met: $6.2 \%(n=29)$. These frequencies did not differ from others described in previous studies conducted in White individuals. ${ }^{21}$ Hardy-Weinberg equilibrium was verified for the present population $\left(\chi^{2}=1.05\right.$, d.f. $\left.=2, P=0.59\right)$.

The final sample consisted of 411 individuals for whom all the variables included in the models were available.

\section{Specificity of the impact of childhood adversity on psychotic-like experiences}

We found a main effect of childhood abuse on positive psychoticlike experiences $(\beta=0.16$, s.e. $=0.05, P=0.002)$ and a marginally significant effect of childhood abuse on negative psychotic-like experiences $(\beta=0.11$, s.e. $=0.06, P=0.055)$ (Table 1$)$. Childhood neglect did not have a direct influence on either positive or negative psychotic-like experiences. Furthermore, no main effect was found for the BDNF-Val66Met polymorphism on either dimension of psychotic-like experiences.

\section{Gene-environment interaction between the BDNF- Val66Met polymorphism and childhood adversity with respect to subsequent psychotic-like experiences}

A significant gene-environment interaction was detected between BDNF Met carriers and childhood abuse with regard to positive psychotic-like experiences $(\beta=0.27$, s.e. $=0.10, P=0.004)$. In this 
Table 1 Main effects of childhood abuse, childhood neglect and the BDNF-Val66Met polymorphism (Val/Val V. Met carriers) on positive and negative psychotic-like experiences, correcting for age, gender, schizotypal personality, cannabis use and trait anxiety

\begin{tabular}{|c|c|c|c|c|c|c|}
\hline & \multicolumn{3}{|c|}{ Positive psychotic-like experiences ${ }^{a}$} & \multicolumn{3}{|c|}{ Negative psychotic-like experiences ${ }^{b}$} \\
\hline & $\beta$ & s.e. & $P$ & $\beta$ & s.e. & $P$ \\
\hline BDNF & -0.385 & 0.358 & 0.282 & 0.338 & 0.409 & 0.409 \\
\hline Childhood abuse & 0.155 & 0.049 & 0.002 & 0.107 & 0.056 & 0.055 \\
\hline Childhood neglect & -0.085 & 0.053 & 0.110 & -0.032 & 0.060 & 0.591 \\
\hline $\begin{array}{l}\text { a. } R^{2}=0.31 \text {. } \\
\text { b. } R^{2}=0.32 \text {. } \\
\text { Values in bold are significant. }\end{array}$ & & & & & & \\
\hline
\end{tabular}

sample, individuals carrying the Met allele had higher scores on adult positive psychotic-like experiences when childhood abuse was present, as compared with participants carrying $\mathrm{Val} / \mathrm{Val}$ homozygotes (Fig. 1). No significant gene-environment interaction was detected with respect to childhood neglect $(\beta=-0.09$, s.e. $=0.05, P=0.110)$.

\section{Discussion}

This study shows that childhood adversity has a strong independent effect on positive psychotic-like experiences and a marginally significant effect on negative psychotic-like experiences, whereas childhood neglect was not associated with either dimension of psychotic-like experiences. The BDNF-Val66Met polymorphism shows a moderating effect between childhood abuse and the later development of positive psychotic-like experiences. These results are not confounded by the effect of gender, age, schizotypal personality, cannabis use or trait anxiety.

\section{Childhood adversity and psychotic-like experiences}

Several years ago it was postulated that light might be shed on the aetiology of psychosis by studying individuals who have psychotic symptoms without being in need of treatment. ${ }^{22}$ Broadly, there are two potential approaches to the measurement of psychotic symptoms in non-clinical samples: one would be to measure schizotypal traits as an attenuated form of psychotic symptoms, whereas the other would involve measuring in the general population the occurrence of those symptoms that are seen in individuals with psychosis. The latter approach assumes that experiencing 'symptoms' of psychosis is not inevitably linked with the clinical disorder. Thus, even though the prevalence of the clinical disorder is low, the prevalence of these 'milder forms' of

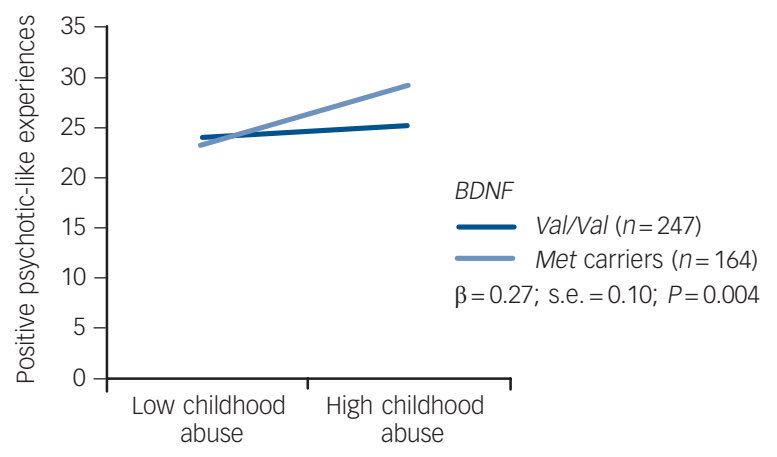

Fig. 1 Graphic respresentation of the interaction effect between childhood abuse and the BDNF-Val66Met polymorphism on positive psychotic-like experiences.

Corrected for age, gender, schizotypal personality, cannabis use and trait anxiety. Exposure to childhood abuse is moderated by the BDNF gene. Met carriers exposed to childhood abuse have significantly higher scores on positive psychotic-like experiences. psychosis, namely psychotic-like experiences, may be much higher. ${ }^{13,22}$ The rate of psychotic-like experiences in the present sample is in line with previous reports. ${ }^{22}$ For example, Barrett \& Etheridge found that $30-40 \%$ of individuals from the general population reported the experience of hearing voices. ${ }^{23}$ Similarly, in a sample of college students, $71 \%$ reported at least brief, occasional hallucinated voices during periods of wakefulness, whereas 39\% reported hearing their thoughts spoken aloud. ${ }^{24}$

Regarding the aetiology of psychotic-like experiences, according to previous research, our findings support the role of childhood adversity as a risk factor underlying the development of psychotic-like experiences in the general population. ${ }^{4-6}$ Specifically, there was a strong association between childhood abuse and positive psychotic-like experiences and a trend towards an association between childhood abuse and negative psychoticlike experiences. These results fit well with recent models suggesting that adverse events, especially those characterised by abuse, may produce a psychological and/or biological vulnerability for the development of positive psychotic symptoms, including subclinical forms such as psychotic-like experiences. ${ }^{22,25,26}$ It has been suggested that early abusive experiences may create an enduring cognitive vulnerability characterised by negative schematic models of the self and the world (for example beliefs about the self as vulnerable to threat, or about others as dangerous) that facilitate external attributions, which may ultimately lead to paranoid ideation. ${ }^{25}$ In this regard, current ideas about the biological consequences of childhood adversity lend even more credibility to the notion of an enduring psychological vulnerability. When exposure to stressors persists, the stressinduced glucocorticoid release can become chronic, leading to permanent changes in the hypothalamic-pituitary-adrenal (HPA) axis. This alteration of HPA functioning can lead to dysregulation of the dopaminergic system, which is generally thought to be involved in psychosis. ${ }^{1,27}$ Specifically, it has been suggested that stress-induced dysregulation of the HPA axis causes increased dopamine receptor densities and greater dopamine release. The dopaminergic system is important as regards the interpretation of stress and threat-related stimuli, and therefore, relevant to the development of positive psychotic symptoms such as paranoid ideation. ${ }^{26}$

In our sample, childhood neglect was not significantly associated with psychotic-like experiences. Although this contrasts with some previous reports, ${ }^{28,29}$ a recent study by Fisher and colleagues $^{30}$ also found no impact of neglect on the expression of psychosis when controlling for the impact of abuse. Conversely, events characterised by abuse have shown the most robust association with psychotic symptoms. ${ }^{6,31,32}$ Moreover, it has been postulated that abusive experiences could have an aetiological significance in psychosis, ${ }^{33}$ and research has described higher rates of abusive maltreatment than neglect among individuals with psychosis. ${ }^{34}$ Hence, it may be that previous associations between psychosis and childhood adversity, where the latter included both abuse and neglect events, were inflated by the effect of abuse. 


\section{BDNF-Val66Met polymorphism, childhood adversity and psychotic-like experiences}

Gene-environment interaction studies have shown exciting findings that made them appear to be promising mechanisms to understanding the joint effect between environmental and genetic factors in the aetiology of complex traits such as psychiatric symptoms. ${ }^{35}$ However, dismissal of gene-environment interaction studies has recently arisen mainly as a result of the failure to replicate; as has happened before with genetic association studies. ${ }^{36}$ It has been argued that the lack of replication may be related to the greater number of potential statistical tests that are possible when interaction effects are included in any analysis, which greatly increases the risk of false positives that can be nominally significant but do not represent true insight. ${ }^{36}$ To prevent this, the present study was developed with a priori hypothesis that guides the choice of the gene and the polymorphism and the environmental risk factor. Furthermore, several reasons were considered to explain why gene-environment interactions might be expected in the relationship between childhood adversity and psychosis. Human development is an environmentally dependent process in which individuals need to adapt to environmental hazards. However, it is implausible that genetic variants do not contribute to individual variation in response to the environment, since this response is associated with preexisting individual differences in temperament, personality and psychophysiology, all of which are known to be under a certain degree of genetic influence. ${ }^{35}$ In this context, one genetic variant that is a candidate for moderating the association between childhood adversity and psychosis is the BDNF-Val66Met polymorphism. This polymorphism consists of a Val/Met single nucleotide polymorphism at position 66 in the BDNF gene, and it has been identified as a functional polymorphism. ${ }^{37}$ The Val variant is associated with higher neuronal BDNF secretory activity than is the Met variant. Additionally, the coexpression of $\mathrm{Val}$ and Met alleles in heterozygotes results in less efficient intracellular trafficking and processing, leading to decreased BDNF secretion. ${ }^{37}$ The secretion of BDNF is crucial for the growth and differentiation of developing neurons in both central and peripheral nervous systems, and BDNF is also implicated in the survival of neuronal cells in response to stress. ${ }^{1,7,37}$ Evidence from animal studies suggests that individuals carrying the Met/Met genotype are more likely to develop anxiety-related behaviours in response to stressful events. ${ }^{7}$ In humans, it has been shown that Met homozygotes and heterozygotes who have experienced childhood adversity could also be more genetically vulnerable to the development of affective symptoms, in comparison to Val homozygotes. ${ }^{8}$ However, the potential moderating effect of the BDNF-Val66Met polymorphism on the relationship between psychosocial stress and psychosis has not been widely explored. To the best of our knowledge, only Simons and colleagues ${ }^{11}$ have studied the relationship between minor stressful daily events, the BDNF-Val66Met polymorphism and paranoid experiences. These authors found that BDNF-Met allele carriers showed more social stress-induced paranoia than did individuals with the $\mathrm{Val} / \mathrm{Val}$ genotype. The present results are in line with these findings. Specifically, we found that the impact of childhood abuse on the development of positive psychotic-like experiences was higher in those individuals carrying the Met allele. This provides evidence of a gene-environment interaction effect, whereby Met carriers would, genetically, be more vulnerable to the effects of childhood abuse than would Val homozygotes.

We believe that these findings are consistent with the hypothesised affective pathway to psychosis, which has been suggested to be preferentially underlying the positive symptoms of psychosis. ${ }^{1}$ As mentioned earlier, childhood adversity has been shown to alter the functioning of the HPA axis, which is one of the most important brain circuits involved in regulating adaptive responses to stress. In this context, the intrusive nature of abusive experiences may indicate that they are especially likely to dysregulate the HPA axis. This dysregulation would, in turn, result in increased dopamine release in mesolimbic brain areas, which has been frequently related to the expression of positive psychotic symptoms. ${ }^{1,2}$

In summary, our results indicate that individuals carrying the Met allele, the variant associated with less BDNF secretion, would be more vulnerable, neurobiologically speaking, to the negative effects of early abusive experiences.

\section{Strengths and limitations}

Among the strengths of the present study it is worth noting that the results were not confounded by the effect of schizotypal personality traits, trait anxiety or cannabis use. Thus, the findings indicate that exposure to childhood abuse increases the risk of reporting adult psychotic-like experiences independently of any pre-existing schizotypal traits, which have also been shown to increase the likelihood of experiencing psychotic symptoms. ${ }^{18}$ Similarly, although the use of cannabis is a well-known environmental risk factor for psychotic-like experiences, ${ }^{38}$ this did not confound the present results as the frequency of cannabis use was controlled for. As regards the inclusion of trait anxiety as a confounder, it has been found that the strong emotions associated with childhood adversity, such as anxiety and memories of the earlier experience, contribute to an increased risk of later psychotic symptoms. ${ }^{39}$ However, as trait anxiety was controlled for, we can rule out the possibility that the occurrence of psychotic-like experiences was linked to the anxiety associated with abusive events experienced in childhood. Overall, the present research design follows the recommendations of a systematic and critical review by Bendall et $a l^{2}$ in that it includes confounders based on previous research into childhood adversity and psychosis.

Despite these strengths, the present study does have a number of limitations. First, the cross-sectional design prevents a robust test of causal associations, although a priori hypotheses were clearly defined and guided all the subsequent analyses as mentioned earlier. Second, the retrospective measure of childhood adversity may constitute an inherent source of bias. That said, the CTQ has been validated and is considered a reliable measure of childhood adversity, ${ }^{15}$ as well as being recommended in the critical review by Bendall and colleagues ${ }^{2}$ as a reliable tool for measuring childhood abuse. Third, although the current findings are in line with those reported by Simons et al, ${ }^{11}$ the studies are not directly comparable since the outcomes and environmental risk factors analysed were different. Studies examining similar hypothesis but that differ in the exact variables analysed or the instruments used to measure such variables can also account for inconsistencies in the results and therefore, failure to replicate. Fourth, although the sample size is similar to that used in previous and similar studies, ${ }^{4,11}$ it can be still considered relatively small. In the light of these limitations, our findings should be considered with caution and need replication in larger samples.

\section{Implications}

Our findings suggest a specific relationship between childhood abuse and positive psychotic-like experiences in the general population. The results also provide evidence for a geneenvironment interaction effect underlying individual behavioural differences in response to childhood abuse; specifically, Met carriers are more likely to report positive psychotic-like experiences in the presence of childhood abuse compared with Val homozygotes. These results now require replication as they may have important implications for future research into the 
aetiological mechanisms operating between childhood adversity and later psychosis.

Silvia Alemany, MSC, Bárbara Arias, PhD, Mari Aguilera, PhD, Unitat d'Antropologia Departament de Biologia Animal, Facultat de Biologia and Institut de Biomedicina (IBUB), Universitat de Barcelona, and Centro de Investigaciones Biomédicas en Red de Salud Mental (CIBERSAM), Instituto de Salud Carlos III, Madrid; Helena Villa, PhD, Jorge Moya, PhD, Manuel I. Ibáñez, PhD, Departament de Psicologia Bàsica, Clínica i Psicobiologia, Facultat de Ciències Humanes i Socials, Universitat Jaume I, Castelló; Helen Vossen, PhD, Unitat d'Antropologia, Departament de Biologia Animal, Facultat de Biologia and Institut de Biomedicina (IBUB), Universitat de Barcelona, and Centro de Investigaciones Biomédicas en Red de Salud Mental (CIBERSAM), Instituto de Salud Carlos III, Madrid; Cristobal Gastó, MD, PhD, Centro de Investigaciones Biomédicas en Red de Salud Mental (CIBERSAM), Instituto de Salud Carlos III, Madrid, and Departamento de Psiquiatría, Instituto Clínico de Neurociencias, Hospital Clínico de Barcelona, and Instituto de Investigaciones Biomédiques August P i sunyer (IDIBAPS), Barcelona; Generós ortet, PhD, Departament de Psicologia Bàsica, Clínica i Psicobiologia, Facultat de Ciències Humanes i Socials, Universitat Jaume I, Castelló; Lourdes Fañanás, BSC, MD, PhD, Unitat d'Antropologia, Departament de Biologia Animal, Facultat de Biologia and Institut de Biomedicina (IBUB), Universitat de Barcelona, and Centro de Investigaciones Biomédicas en Red de Salud Mental (CIBERSAM), Instituto de Salud Carlos III, Madrid, Spain

Correspondence: Lourdes Fañanás, Unitat d'Antropologia, Dep. Biologia Animal, Facultat Biologia, Universitat de Barcelona. Av. Diagonal 645, 08028 Barcelona, Spain. Email: Ifananas@ub.edu

First received 21 Dec 2010, final revision 31 Jan 2011, accepted 21 Mar 2011

\section{Funding}

This study was supported through research projects funded by the Ministry of Science and Innovation (SAF2008-05674-C03-00/03 and PSI2008-05988) and the Institute of Health Carlos III, CIBER of Mental Health (CIBERSAM) and also by the Comissionat per a Universitats Recerca del DIUE of the Generalitat de Catalunya (2009SGR827). S.A. thanks the Institute of Health Carlos III for her PhD grant (FI00272).

\section{References}

1 Van Winkel R, Stefanis NC, Myin-Germeys I. Psychosocial stress and psychosis. A review of the neurobiological mechanisms and the evidence for gene-stress interaction. Schizophr Bull 2008; 34: 1095-105.

2 Bendall S, Jackson HJ, Hulbert CA, McGorry PD. Childhood trauma and psychotic disorders: a systematic, critical review of the evidence. Schizophr Bull 2008; 34: 568-79.

3 Bebbington PE, Bhugra D, Brugha $T$, singleton N, Farrell $M$, Jenkins $R$, et al. Psychosis, victimisation and childhood disadvantage. Evidence from the second British National Survey of Psychiatric Morbidity. Br J Psychiatry 2004; 185: 220-6.

4 Kelleher I, Harley M, Lynch F, Arseneault L, Fitzpatrick C, Cannon M. Associations between childhood trauma, bullying and psychotic symptoms among a school-based adolescent sample. Br J Psychiatry 2008; 193: 378-82.

5 Spauwen J, Krabbendam L, Lieb R, Wittchen HU, van Os J. Impact of psychological trauma on the development of psychotic symptoms: relationship with psychosis proneness. Br J Psychiatry 2006; 188: 527-33.

6 Janssen I, Krabbendam L, Bak M, Hanssen M, Vollebergh W, de Graaf R, et al. Childhood abuse as a risk factor for psychotic experiences. Acta Psychiatr Scand 2004; 109: 38-45.

7 Chen ZY, Jing D, Bath KG, Ieraci A, Khan T, Siao CJ, et al. Genetic variant BDNF (Val66Met) polymorphism alters anxiety-related behavior. Science 2006; 314: 140-3.

8 Aguilera M, Arias B, Wichers M, Barrantes-Vidal N, Moya J, Villa H, et al. Early adversity and 5-HTT/BDNF genes: new evidence of gene-environment interactions on depressive symptoms in a general population. Psychol Med 2009; 39: 1425-32.

9 Buckley PF, Mahadik S, Pillai A, Terry Jr A. Neurotrophins and schizophrenia Schizophr Res 2007; 94: 1-11.

10 van OS JS, Sham P. Gene-environment interactions. In The Epidemiology of Schizophrenia (eds RJ Murray, PB Jones, E Susser, J van Os, M Cannon): 235-53. Cambridge University Press, 2003.

11 Simons CJ, Wichers M, Derom C, Thiery E, Myin-Germeys I, Krabbendam L, et al. Subtle gene-environment interactions driving paranoia in daily life. Genes Brain Behav 2009; 8: 5-12.

12 Freedman ML, Reich D, Penney KL, McDonald GJ, Mignault AA, Patterson N et al. Assessing the impact of population stratification on genetic association studies. Nat Genet 2004; 36: 388-93.

13 Stefanis NC, Hanssen M, Smirnis NK, Avramopoulos DA, Evdokimidis IK, Stefanis $\mathrm{CN}$, et al. Evidence that three dimensions of psychosis have a distribution in the general population. Psychol Med 2002; 32: 347-58.
14 Bernstein DPFL. Childhood Trauma Questionnaire: A Retrospective Self-report. The Psychological Corporation, 1998.

15 Bernstein DP, Stein JA, Newcomb MD, Walker E, Pogge D, Ahluvalia T, et al. Development and validation of a brief screening version of the Childhood Trauma Questionnaire. Child Abuse Negl 2003; 27: 169-90.

16 Barkus $\mathrm{E}$, Lewis S. Schizotypy and psychosis-like experiences from recreational cannabis in a non-clinical sample. Psychol Med 2008; 38: 1267-76.

17 Freeman $D$, McManus S, Brugha $T$, Meltzer $H$, Jenkins R, Bebbington $P$. Concomitants of paranoia in the general population. Psychol Med 2010; 24: 1-14.

18 Barrantes-Vidal N, Lewandowski KE, Kwapil TR. Psychopathology, social adjustment and personality correlates of schizotypy clusters in a large nonclinical sample. Schizophr Res 2010; 122: 219-25.

19 Raine $A B$, Benishay D. A brief screening instrument for schizotypal personality disorder. J Pers Disord 1995; 9: 346-55.

20 Spielberg CG, Gorsuch RL, Lushene RE. STAI Manual for the State-Trait Anxiety Inventory. Consulting Psychologists Press, 1970.

21 Egan MF, Kojima M, Callicott JH, Goldberg TE, Kolachana BS, Bertolino A, et al. The BDNF val66met polymorphism affects activity-dependent secretion of BDNF and human memory and hippocampal function. Cell 2003; 112 : 257-69.

22 Johns LC, van Os J. The continuity of psychotic experiences in the general population. Clin Psychol Rev 2001; 21: 1125-41.

23 Barrett TE, Etheridge JB. Verbal hallucinations in normals. I: People who hear voices. Appl Cogn Psychol 1992; 6: 379-87.

24 Posey TL, Losch ME. Auditory hallucinations of hearing voices in 375 normal subjects. Imagination Cognition Pers 1983; 57: 99-113.

25 Garety PA, Kuipers E, Fowler D, Freeman D, Bebbington PE. A cognitive model of the positive symptoms of psychosis. Psychol Med 2001; 31: 189-95.

26 Read J, Perry BD, Moskowitz A, Connolly J. The contribution of early traumatic events to schizophrenia in some patients: a traumagenic neurodevelopmental model. Psychiatry 2001; 64: 319-45.

27 Heim C, Newport DJ, Heit S, Graham YP, Wilcox M, Bonsall R, et al. Pituitary-adrenal and autonomic responses to stress in women after sexual and physical abuse in childhood. JAMA 2000; 284: 592-7.

28 Vogel M, Spitzer C, Kuwert P, Moller B, Freyberger HJ, Grabe HJ. Association of childhood neglect with adult dissociation in schizophrenic inpatients. Psychopathology 2009; 42: 124-30.

29 Gil A, Gama CS, de Jesus DR, Lobato MI, Zimmer M, Belmonte-de-Abreu P. The association of child abuse and neglect with adult disability in schizophrenia and the prominent role of physical neglect. Child Abuse Negl 2009; 33: 618-24.

30 Fisher $\mathrm{HL}$, Jones PB, Fearon $\mathrm{P}$, Craig TK, Dazzan $\mathrm{P}$, Morgan $\mathrm{K}$, et al. The varying impact of type, timing and frequency of exposure to childhood adversity on its association with adult psychotic disorder. Psychol Med 2010; 24: 1-12.

31 Whitfield CL, Dube SR, Felitti VJ, Anda RF. Adverse childhood experiences and hallucinations. Child Abuse Negl 2005; 29: 797-810.

32 Morgan C, Fisher H. Environment and schizophrenia: environmental factors in schizophrenia: childhood trauma-a critical review. Schizophr Bull 2007; 33: 3-10.

33 Harris T. Recent developments in the study of life events in relation to psychiatric and physical disorders. In Psychiatric Epidemiology: Progress and Prospects (ed B Cooper): 81-102. Croom Helm, 1987.

34 Hlastala SA, Mcclellan J. Phenomenology and diagnostic stability of youths with atypical psychotic symptoms. J Child Adolesc Psychopharmacol 2005; 15: 497-509.

35 Rutter M, Moffitt TE, Caspi A. Gene-environment interplay and psychopathology: multiple varieties but real effects. J Child Psychol Psychiatry 2006; 47: 226-61.

36 Munafo MR, Flint J. Replication and heterogeneity in gene $\times$ environment interaction studies. Int J Neuropsychopharmacol 2009; 12: 727-9.

37 Chen ZY, Patel PD, Sant G, Meng CX, Teng KK, Hempstead BL, et al. Variant brain-derived neurotrophic factor (BDNF) (Met66) alters the intracellular trafficking and activity-dependent secretion of wild-type BDNF in neurosecretory cells and cortical neurons. J Neurosci 2004; 24: 4401-11.

38 Arseneault L, Cannon M, Witton J, Murray RM. Causal association between cannabis and psychosis: examination of the evidence. Br J Psychiatry 2004; 184: 110-7.

39 Freeman D, Garety PA. Connecting neurosis and psychosis: the direct influence of emotion on delusions and hallucinations. Behav Res Ther 2003; 41: 923-47. 